Rev Biomed 2004; 15:57- 68.

\title{
La contribución de la Antropología Médica al estudio comparativo de la cultura en salud: el caso del sistema frío/calor en los pacientes con afecciones respiratorias.
}

\author{
Antropología Médica
}

Javier E García de Alba-García ${ }^{1}$, Christine von Glascoe ${ }^{2}$, Arthur J Rubel ${ }^{3}$, Carmella C Moore ${ }^{3}$, Silvia Casasola $^{3}$, Mario Marquez Amezcua ${ }^{4}$.

${ }^{1}$ UISESS- IMSS-Jalisco /HCG. J.I.Menchaca. ${ }^{2}$ El Colegio de la Frontera Norte. ${ }^{3}$ Universidad de California-Irvine, E.E.U.U. ${ }^{4}$ Secretaria de Salud-Jalisco, México.

\section{RESUMEN.}

Objetivo. Analizar el sistema humoral frío/calor para seis enfermedades respiratorias en un grupo formado por pacientes de los Centros de Salud 1 y 3 de Guadalajara y otro del Centro de Salud de Ensenada, para establecer diferencias y concordancias sobre conocimientos culturales.

Material y Métodos. Estudio transversal, comparativo realizado en 1997, durante el cual se aplicó un cuestionario estructurado a 362 pacientes mexicanos, sobre la condición humoral frío/calor en el catarro común, neumonía, bronquitis, gripa, tuberculosis y asma.

Resultados. La atribución humoral sobre frío/calor sobre seis enfermedades respiratorias, sigue vigente, en las personas estudiadas de Guadalajara y Ensenada. El porcentaje de los entrevistados ¿Qué contestaron afirmativamente? Varió respectivamente entre 53\% y $69 \%$ y $56 \%$ y $81 \%$, respectivamente.

Las mujeres tuvieron un acuerdo más alto y consistente respecto al genero masculino, quienes muestran un acuerdo diferente a la mujer en TB.

Conclusiones. El sistema humoral, frío/calor continúa vigente en la población estudiada para las 6 enfermedades respiratorias estudiadas, en función de su difusión espacial y diferenciación por genero.

(Rev Biomed 2004; 15:57- 68)

Palabras clave: Enfermedades respiratorias, Antropología Médica, frío/calor, género, México.

\section{SUMMARY.}

The contribution of Medical Anthropology in the comparative study of health culture: the case of the hot/cold system in patients with respiratory diseases.

Objective. To analyze the humoral system of HotCold for six respiratory diseases, in two groups of patients at the Public Health Clinics 1 and 3 of Guadalajara and Ensenada, to establish differences and agreement on cultural knowledge.

Solicitud de sobretiros: Dr. Javier E. García de Alba García. Unidad de Investigación Social, Epidemiológica y de Servicios de Salud. (Bajos de la Farmacia Central) Centro Médico de Occidente del IMSS. Belisario Domínguez 1000, C.P. 44340, Guadalajara, Jalisco, México. Correo electrónico: javier_91046@yahoo.com

Recibido el 18/Septiembre/2003. Aceptado para publicación 9/Marzo/2004.

Este artículo está disponible en http://www.uady.mx/sitios/biomedic/revbiomed/pdf/rb041518.pdf 


\section{JE García de Alba G, C von Glascoe, AJ Rubel, CC Moore, S Casasola, M Marquez-Amezcua.}

Material and Methods. A cross sectional comparative study was conducted in 1997, using structured questionnaire. It was administered to 362 Mexicans patients, concerning the Hot-Cold humoral condition of the common cold, pneumonia, bronchitis, influenza, tuberculosis and asthma.

Results. Humoral quality of cold/hot to describe six respiratory diseases is still in use in Guadalajara and Ensenada. The percentage of informants with affirmative answers varied from $53 \%$ to $69 \%$ and $56 \%$ to $81 \%$ respectively. Women showed a higher and more consistent agreement than men, the biggest difference being in their assignment of humoral quality to $\mathrm{TB}$.

Conclusions. The humoral system of hot/cold is still being used by the population for the six diseases studied, in function of its spatial diffusion and differentiation by gender. (Rev Biomed 2004; 15:57-68)

Key words: Respiratory diseases, Medical Anthropology, cold/hot, gender, México.

\section{INTRODUCCIÓN.}

Uno de los problemas que esta enfrentando el sistema de atención médica actual es la necesidad de desarrollar un enfoque integral que tome en cuenta la perspectiva del paciente, para realizar una práctica médica comprensiva de calidad y con calidez. Conocer los valores, las tradiciones, el significado y el sentido que dan los pacientes a sus padecimientos, ha resultado de singular importancia, en la aplicación exitosa de programas de atención médica sanitaria. Para tal efecto la antropología médica ha sido el vehículo disciplinar para obtener conocimiento sobre este tipo de problemas (1).

Nuestro trabajo alude a las enfermedades respiratorias agudas, afecciones que en el mundo, representan el $7 \%$ de la mortalidad general (2).

En México, la tasa de mortalidad por enfermedades respiratorias, incluidas neumonías e influenza, disminuyó de 46 defunciones 100,000 habitantes en 1997 a 43 por 100,000 en 1999, que significó pasar del séptimo al octavo lugar de las principales causas de defunción. Paradójicamente, las consultas médicas registradas por enfermedades respiratorias agudas se incrementaron de 25.999 por 100,000 consultas en 1997 a 36,876 en 2000 (3).

Los datos anteriores, nos indican que las enfermedades respiratorias, continúan siendo un problema de salud mundial y nacional.

Este problema en la práctica se hace más complejo, porque de acuerdo a la historia natural de la enfermedad, muchos de los padecimientos respiratorios en su fase inicial, clínicamente, son indistinguibles unos de otros, debiéndose recurrir a técnicas paraclínicas de tamizaje, para efectuar diagnostico y tratamiento temprano de algunos de ellos; un ejemplo sería el caso de la tuberculosis pulmonar en los pacientes que con más de 4 semanas de manifestar tos, acuden para su atención a la consulta externa de las instituciones de salud (4).

Si bien las enfermedades respiratorias han sido estudiadas desde puntos de vista clínico y epidemiológico, este problema no ha sido abordado como padecimientos desde el punto de vista antropológico (5-8), en donde se tomen en cuenta las creencias del paciente, considerándolas de una forma amplia, es decir, como un complejo sistema cultural, que condiciona conductas cotidianas donde se incluye al proceso salud-enfermedad-atención.

El presente trabajo analiza el sistema frío/calor en seis enfermedades respiratorias: el catarro común, la neumonía, la bronquitis, la gripa, la tuberculosis y el asma, que fueron seleccionadas a partir de los conocimientos culturales que poseen los pacientes sobre los problemas que afectan las vías respiratorias, en dos localidades mexicanas, con el propósito de:

1.- Determinar el nivel de acuerdo del conocimiento sobre las cualidades humorales de frío/ calor de 6 enfermedades respiratorias en pacientes de los estratos populares, que asisten a los Centros de Salud seleccionados.

2.- Determinar diferencias en el nivel de acuerdo cultural sobre las cualidades humorales de frío/calor de 6 enfermedades respiratorias, en pacientes entre los géneros estudiados.

Visto el sistema humoral frío/calor como

\section{Revista Biomédica}




\section{Sistema frío/calor en los pacientes con afecciones respiratorias.}

características de los seis padecimientos considerados, las cuales forman parte de marcos explicativos que van más allá de la simple dicotomía frío/calor, ya que la "cualidad" humoral, a decir de Foster, sirve entre otras cosas como un lazo de unión entre el modo de vida, los padecimientos y un dominio cultural mayor, como lo es el sistema médico de los grupos sociales afectados (9).

\section{MATERIAL Y MÉTODOS.}

Se realizó una investigación con enfoque epidemiológico y antropológico, mediante un diseño transversal y comparativo durante el verano del año de 1997 en las ciudades de Guadalajara y Ensenada, en función de su importancia regional como centros de desarrollo económico y poblacional.

Como paso inicial, se entrevistaron todos los pacientes mayores de 15 años de edad, asistentes por primera vez a la consulta externa, en los Centros de Salud 1 y 3 de Guadalajara y de Ensenada, durante una semana, aplicándoseles la técnica de listas libres, preguntándoseles: Dígame ¿cuales son las principales enfermedades respiratorias por las que acude a este Centro de Salud?

De las listas obtenidas, el catarro común, la neumonía, la bronquitis, la gripa, la tuberculosis y el asma, cubrieron el mayor porcentaje (95\%) de los padecimientos enlistados, por lo que respetándose esta nomenclatura señalada, fueron seleccionados para su estudio.

Quince días después de la aplicación de las listas libres, durante una semana, en todos los pacientes mayores de 15 años, de primera vez, con signos y síntomas respiratorios, que se presentaron a la consulta externa del Centro de Salud \#1 de Guadalajara y del Centro de Salud de Ensenada, antes de pasar con el médico, se les aplicó un cuestionario semi estructurado, diseñado para explorar la estructura semántica de conceptos compartidos, como conocimiento cultural obtenido por consenso por dichos pacientes.

El cuestionario que versaba sobre los seis padecimientos respiratorios seleccionados, incluyó la cualidad humoral frío/calor y se basó en el modelo explicativo de Kleimnan (10) de motivos, tratamiento e implicaciones de los seis padecimientos en cuestión. El instrumento se estructuró y piloteó, con preguntas culturalmente apropiadas que pudieran ser cuantificadas para su análisis (11).

Por ejemplo: En el caso de la tuberculosis en la estructuración de las preguntas, se partió de supuestos de que ésta es una enfermedad con signos y síntomas circunscritos y constantes como la tos, el esputo hemoptoico, el sudor nocturno y la pérdida de peso.

Respecto a la validez y limitaciones del diseño utilizado conviene hacer notar que:

1.- Las poblaciones usuarias de unidades de salud, si bien plantean un sesgo de selección a favor de la aceptación del modelo de tratamiento biomédico, este sesgo se puede objetivar al formar grupos de comparación que pertenezcan al mismo tipo de unidad, pero en diferente espacios; es decir que los participantes estén afectados en la misma dirección, para que los hallazgos no se vean afectados de manera adversa.

2.- Los informantes fueron del mismo estrato popular bajo (población abierta de trabajadores sin seguridad social) tanto en Guadalajara como en Ensenada.

3.- Para evitar otros sesgos de selección o históricos los participantes fueron entrevistados los mismos días y en horarios similares.

4.- Los grupos fueron mutuamente contrastados al analizar las construcciones culturales por lugar de residencia, edad, genero y nivel de escolaridad, al mismo tiempo que se controlaban otras variables, al equiparar nivel socio económico, accesibilidad a la atención que incluyen transporte, otros costos de traslados y dificultades de interacción con el equipo de salud (12).

El proceso estadístico de datos se realizo con apoyo de los programas: Epi Info 6.4 y Statgraphics 5.0, utilizando como pruebas inferenciales: el análisis de varianza para datos comparativos de muestras en escala de intervalo, la Ji cuadrada para cuadros 2 por 2, con datos en escala ordinal o nominal y la prueba binomial con dos colas para comparación de los porcentajes de una misma muestra, basada en la 
JE García de Alba G, C von Glascoe, AJ Rubel, CC Moore, S Casasola, M Marquez-Amezcua.

Cuadro 1

Distribución de pacientes con enfermedad respiratoria por lugar de residencia y género.

\begin{tabular}{lccc}
\hline \multirow{2}{*}{ Lugar de Residencia } & \multicolumn{2}{c}{ Género del Paciente } & \multirow{2}{*}{ Total } \\
& Masculino & Femenino & \\
\hline Ensenada & $67(49 \%)$ & $68(51 \%)$ & 135 \\
Guadalajara & $79(35 \%)$ & $148(65 \%)$ & 227 \\
Totales & $146(40 \%)$ & $216(60 \%)$ & 362 \\
\hline
\end{tabular}

Fuente: Cuestionario semi- estructurado

Análisis de Varianza, Lugar de residencia y Género: $F=0.029, p=0.866$

hipótesis de no acuerdo o sea de una distribución de $50 \%$ de respuestas afirmativas y $50 \%$ de respuestas negativas a una pregunta especifica. En todos los casos se consideró como significativa una " $\mathrm{p}$ " igual o menor de 0.05 y cuando la p fue menor que una diezmilésima se anotó sólo $\mathrm{p}<0.001$.

El objetivo de nuestro estudio fue analizar el sistema humoral frío/calor para seis enfermedades respiratorias en un grupo formado por pacientes de los Centros de Salud 1 y 3 de Guadalajara y otro del Centro de Salud de Ensenada, para establecer diferencias y concordancias sobre conocimientos culturales.

\section{RESULTADOS.}

Al efecto se entrevistaron con un cuestionario semi- estructurado 362 pacientes (135 en Ensenada y 227 en Guadalajara), de los cuales en Ensenada 67 fueron hombres y 68 mujeres; para Guadalajara 79 fueron hombres y 148 mujeres ( $>0.05$ ). Siendo el promedio de edad de la muestra de Ensenada 8 años más joven que la de Guadalajara ( $\mathrm{p}<0.001)$ y la de
Guadalajara con menor nivel de escolaridad que Ensenada $(p<0.001)$ (cuadros 1-3).

En los cuadros 4 y 5 se observa que en las muestras de ambas ciudades, un mayor nivel de acuerdo para considerar frías las condiciones respiratorias estudiadas. En otras palabras, más de la mitad de los entrevistados evidencian compartir un mismo sistema de conocimientos sobre el aspecto humoral de las afecciones estudiadas.

Nótese que los mayores porcentaje de acuerdo sobre la condición humoral fría se ubican en los padecimientos de mayor incidencia en la población $(13,14)$.

Los Cuadros 6 y 6-a, ponen de manifiesto la asociación entre el género femenino y el acuerdo sobre la cualidad humoral fría con respecto al catarro, la neumonía y la bronquitis comparadas con los varones ( $82 \%, 78 \%$ y $76 \%$ vs, $71 \%, 61 \%$ y $66 \%)$. En las otras tres enfermedades: gripe, asma y tuberculosis el acuerdo sobre su cualidad fría, señalado por las mujeres fue más consistente que en los hombres. Los varones, no mostraron acuerdo sistemático sobre la

Cuadro 2

Promedio y rango de edades en pacientes con enfermedad respiratoria por lugar de residencia y género.

\begin{tabular}{lccc}
\hline Lugar de Residencia & $\begin{array}{c}\text { Género del Paciente (promedio y rango) } \\
\text { Masculino }\end{array}$ & Femenino & Total \\
\hline Ensenada & $31.1(17-70)$ & $34.1(11-91)$ & $32.6(11-91)$ \\
Guadalajara & $39.3(14-78)$ & $41.7(15-87)$ & $40.9(14-87)$ \\
\hline
\end{tabular}

Fuente: Cuestionario semi- estructurado

Análisis de Varianza de la Edad por Lugar y Genero.

Lugar: $\mathrm{F}=17.7, \mathrm{p}<0.001$ Género: $\mathrm{F}=2.07, \mathrm{p}=0.151$

\section{Revista Biomédica}


Sistema frí/calor en los pacientes con afecciones respiratorias.

Cuadro3

Distribución de pacientes con enfermedad respiratoria por lugar de residencia, género y escolaridad.

\begin{tabular}{|c|c|c|c|c|c|c|c|}
\hline \multirow{4}{*}{$\begin{array}{l}\text { Escolaridad } \\
\text { Formal } \\
\text { Ninguna }\end{array}$} & \multicolumn{7}{|c|}{ Lugar de Residencia y Género } \\
\hline & \multicolumn{3}{|c|}{ Ensenada } & \multicolumn{4}{|c|}{ Guadalajara } \\
\hline & Masculino & \multicolumn{2}{|c|}{ Femenino } & \multicolumn{2}{|c|}{ Masculino } & \multicolumn{2}{|c|}{ Femenino } \\
\hline & $10 \%$ & 7 & $11 \%$ & 16 & $20 \%$ & 25 & $17 \%$ \\
\hline 1 a 3 años & $17 \%$ & 5 & $7 \%$ & 20 & $25 \%$ & 36 & $24 \%$ \\
\hline 4 a 6 años & $25 \%$ & 24 & $36 \%$ & 22 & $29 \%$ & 65 & $44 \%$ \\
\hline 7 - 9 años & $42 \%$ & 27 & $39 \%$ & 16 & $20 \%$ & 16 & $11 \%$ \\
\hline 10 o más & $46 \%$ & & $7 \%$ & 5 & $6 \%$ & 6 & $4 \%$ \\
\hline Totales & $67100 \%$ & 68 & $100 \%$ & 79 & $100 \%$ & 148 & $100 \%$ \\
\hline
\end{tabular}

Fuente: Cuestionario semi- estructurado

Guadalajara $\mathrm{Ji}^{2}=7.62$, g de $1: 4 \mathrm{p}=0.10$

Ensenada $\quad \mathrm{Ji}^{2}=3.57, \mathrm{~g}$ de $1: 4 \mathrm{p}=0.46$

cualidad humoral fría de la tuberculosis.

El género femenino presentó con significancia $(\mathrm{p}<0,05)$ mayores porcentajes de respuestas afirmativas sobre la cualidad fría de los problemas respiratorios, con excepción de la bronquitis $(\mathrm{p}=0.09)$ (ver cuadro 6 y 6a).

En el cuadro 7 se muestra como este conocimiento cultural sobre la cualidad humoral fría de los padecimientos respiratorios estudiados, es consistente y que prácticamente no fue influenciada por la edad de las personas analizadas en ambas muestras. La excepción en este cuadro, es la gripa $(\mathrm{p}=0.04)$ en la muestra de Ensenada, donde en los jóvenes hay menos acuerdo sobre la cualidad fría del padecimiento.
En el cuadro 8, se observa, en las muestras de las dos ciudades, que independientemente de la escolaridad, el acuerdo sobre la condición humoral fría se mantiene coherente y sin diferencias estadísticamente significativas (con excepción de la bronquitis ( $p=0.03$ ) en la muestra de Ensenada en los pacientes de menor escolaridad).

\section{DISCUSIÓN.}

Los primeros etnógrafos como Rivers, Evans Pritchard y Malinosky a partir de sus inquietudes y enfoques teórico-metodológicos, reportaron hallazgos que han servido como espejo a la humanidad, para comprender nuestras normas, valores, tradiciones e instituciones, como parte de la cultura desarrollada

Cuadro 4

Cualidad humoral de padecimientos respiratorios seleccionados según lugar de residencia*

\begin{tabular}{lccc}
\hline \multirow{2}{*}{ Padecimiento } & \multicolumn{2}{c}{ ENSENADA, BAJA CALIFORNIA NORTE } & \\
& Número de Respuestas* & \%Frío / \% Caliente & $\mathrm{p}$ \\
\hline Catarro Común & 109 & $78 / 22$ & $<0.001$ \\
Neumonía & 99 & $74 / 26$ & $<0.001$ \\
Bronquitis & 98 & $73 / 27$ & $<0.001$ \\
Gripa & 108 & $70 / 30$ & $<0.001$ \\
Tuberculosis & 75 & $69 / 31$ & 0,001 \\
Asma & 81 & $65 / 35$ & 0.006 \\
\hline
\end{tabular}

Fuente: Cuestionario semi- estructurado

* Basadas en las respuestas afirmativas o negativas de los 135 pacientes a la pregunta ¿Es la enfermedad X caliente o fría? 
JE García de Alba G, C von Glascoe, AJ Rubel, CC Moore, S Casasola, M Marquez-Amezcua.

Cuadro 5

Cualidad humoral de padecimientos respiratorios seleccionados según lugar de residencia*

\begin{tabular}{lccc}
\hline \multirow{2}{*}{ Padecimiento } & \multicolumn{2}{c}{ GUADALAJARA, JALISCO } & \\
& Número de Respuestas* & \%Frío / \% Caliente & $\mathrm{p}$ \\
\hline Catarro Común & 157 & $77 / 23$ & $<0.001$ \\
Bronquitis & 155 & $72 / 28$ & $<0.001$ \\
Asma & 131 & $71 / 29$ & $<0.001$ \\
Neumonía & 152 & $68 / 32$ & $<0.001$ \\
Gripa & 158 & $65 / 35$ & 0,001 \\
Tuberculosis & 121 & $60 / 40$ & 0.006 \\
\hline
\end{tabular}

Fuente: Cuestionario semi- estructurado

* Basadas en las respuestas afirmativas o negativas de los 227 pacientes a la pregunta ¿Es la enfermedad X caliente o fría?

por el ser humano. Dentro de este enfoque, los estudios comparativos han sido recibidos con entusiasmo, debido a la posibilidad de sugerir hipótesis con base en los resultados encontrados, al estudiar diversas sociedades $(15,16)$. Al efecto, en las enfermedades respiratorias, juegan un papel importante para su diagnóstico los tratamientos oportunos, aspectos culturales como la tradición, las costumbres, los valores, etc. Y debido a que cada grupo social le otorga un sentido y un significado particular a cada afección respiratoria, a manera de padecimiento, una manera de abordar estos problemas de salud, es precisamente a través de estudios comparativos de índole inter e intra cultural que ayudan a consolidar o no hipótesis de trabajo en antropología médica.

Las características humorales de los padecimientos forman parte de esos marcos explicativos que van más allá de la simple dicotomía frío/calor, ya que la "cualidad" humoral, a decir de Foster, sirve entre otras cosas como un lazo de unión entre la cotidianidad de los sujetos, los padecimientos que sufren y dominios culturales mayores, como lo es el sistema médico de atención de estos sujetos sociales (9) .

Se sabe que el sub-sistema humoral dicotómico del frío-calor, esta ligado estrechamente al equilibrio de la salud-enfermedad-atención. Sin embargo, su origen conceptual ha suscitado polémica e interesantes comentarios entre diversos autores, aludiendo unos, como Foster (9), su origen Hipocrático, producto de la degeneración de la teoría de los cuatro humores en sólo dos, posición que se ha denominado prodifusionista y que ha sido adoptada por numerosos antropólogos médicos (17). Otros investigadores,

\section{Cuadro 6}

Cualidad humoral de padecimientos respiratorios seleccionadas en las mujeres*

\begin{tabular}{lccc}
\hline Padecimiento & No de Respuestas & $\begin{array}{c}\text { MUJERES } \\
\% \text { Frío / \% Caliente }\end{array}$ \\
\hline Catarro común & 154 & $82 \% / 18 \%$ & $\mathrm{p}$ \\
Neumonía & 149 & $78 \% / 22 \%$ & $<0.001$ \\
Bronquitis & 158 & $76 \% / 24 \%$ & $<0.001$ \\
Asma & 127 & $76 \% / 24 \%$ & $<0.001$ \\
Tuberculosis & 111 & $74 \% / 26 \%$ & $<0.001$ \\
Gripa & 158 & $73 \% / 27 \%$ & $<0.001$ \\
\hline
\end{tabular}

Fuente: Cuestionario semi- estructurado

* Basadas en las respuestas afirmativas o negativas de 216 mujeres estudiadas, a la pregunta ¿Es la enfermedad X caliente o fría?

\section{Revista Biomédica}


Sistema frío/calor en los pacientes con afecciones respiratorias.

Cuadro 6-a

Cualidad humoral de padecimientos respiratorios seleccionadas en los varones*

\begin{tabular}{lccl}
\hline & \multicolumn{2}{c}{ VARONES } & \\
Padecimiento & No de Respuestas & $\%$ Frío / \% Caliente & $\mathrm{p}$ \\
\hline Catarro Común & 112 & $71 \% / 29 \%$ & $<0.001$ \\
Bronquitis & 95 & $66 \% / 34 \%$ & 0.0019 \\
Neumonía & 102 & $61 \% / 39 \%$ & 0.0375 \\
Gripa & 108 & $58 \% / 42 \%$ & 0.1018 \\
Asma & 85 & $58 \% / 42 \%$ & 0.1927 \\
Tuberculosis & 85 & $49 \% / 51 \%$ & $\sim 1.0000$. \\
\hline
\end{tabular}

Fuente: Cuestionario semi- estructurado

* Basadas en las respuestas afirmativas o negativas de 146 varones estudiados, a la pregunta

¿Es la enfermedad X caliente o fría?

Nota: Las diferencias porcentuales de respuestas afirmativas entre mujeres y varones (cuadros 6 y 6 a) para las enfermedades analizadas Catarro (82 vs 71), Neumonía (78 vs 61), Bronquitis (76 vs 66), Asma (76 vs 58), Tuberculosis (74 vs 49) y Gripa (73 vs 58) presentaron respectivamente los siguientes valores: Ji cuadrada $=4.01$ y $\mathrm{p}=0.04 ;$ Ji cuadrada $=8.55$ y $\mathrm{p}=0.003$; Ji cuadrada $=2.75$ y $\mathrm{p}=0.09 ; \mathrm{Ji}$ cuadrada $=8.83$ y $\mathrm{p}=0.03 ; \mathrm{Ji}$ cuadrada $=12.39$ y $\mathrm{p}=0.0004 ; \mathrm{Ji}$ cuadrada $=6.05$ y $\mathrm{p}=0.013$

como López Austin (18), preconizan la génesis americana del sistema, insistiendo que esta dicotomía, va más allá de una forma de clasificación de remedios, enfermedades y alimentos $\dagger$.

Desde el punto de vista de la tradición, algunos investigadores $(9,19-21)$ han reportado una perdida significativa de acuerdo compartido en las personas sobre el conocimiento del sistema humoral frio/calor, sugiriendo algunos que dicho conocimiento cultural nunca haya sido importante o que se esté atenuando por la medicalización del concepto salud-enfermedadatención; o que la gente lo haya olvidado o descontinuado en sus componentes esenciales; o/y que la gente joven haya perdido la capacidad de aprender y/o retener este conocimiento tradicional.

Sin embargo, nuestros resultados, muestran que más de la mitad de los pacientes entrevistados relacionan la enfermedad respiratoria con su cualidad humoral fría, tanto en Guadalajara como en Ensenada, por que suponemos sugieren:

1.- Que el enfoque humoral continúa siendo vigente en la cultura de los estratos populares que asisten a los centros de salud, donde fueron estudiados

2.- Que el sistema continúa siendo prevaleciendo de manera mayoritaria en dichos estratos

3.- Que no es una simple dicotomía, y que diferentes factores están involucrados en la explicación de este paradigma (como son la edad, el género y el lugar de residencia).

Es decir, que el sub-sistema frío/calor, continúa enraizado en la vida cotidiana de nuestros informantes de estratos populares, independientemente de su edad, escolaridad y la ubicación espacial. Lo cual pone de manifiesto no tan sólo su actualidad, amplitud y complejidad, sino también la necesidad de incluirlo, en la atención integral y comprensiva del problema de las afecciones respiratorias, principalmente en las prácticas sanitarias asistenciales donde se involucra la educación en salud y la educación para la salud.

En relación al supuesto de la vigencia del conocimiento sobre las cualidades humorales frías en la mayoría de las enfermedades respiratorias

\footnotetext{
$†$ Existen otras posiciones como la de Bastien y Shaedel (22) y Ackerknecht (23) que suponen un origen andino de la dicotomía frío / calor. Madsen y Orso (cf: Hahold), (24) la consideran un sincretismo entre la medicina académica española y los principios de dualidad indígenas. Para otros el origen actualmente no tiene relevancia, ya que el enraizamiento en el diario acontecer es suficiente para considerar estos principios humorales como indígenas. Por otra parte Kuschick (25) señala que "hasta ahora se ha logrado demostrar precisamente con poco éxito en la medicina popular española el vínculo entre la medicina humoral académica y la popular en la Edad Media tardía ya principios de la Edad Moderna", además de indicar que el sentido de frío / caliente en la medicina popular española, es efecto del poder y no de los atributos caliente-desecador o frío-húmedo.
} 
JE García de Alba G, C von Glascoe, AJ Rubel, CC Moore, S Casasola, M Marquez-Amezcua.

Cuadro 7

Cualidad humoral de padecimientos respiratorios seleccionados por edad encima o debajo del promedio

\begin{tabular}{|c|c|c|c|c|}
\hline \multirow[t]{2}{*}{ GRIPA } & \multicolumn{2}{|c|}{ Ensenada } & \multicolumn{2}{|c|}{ Guadalajara } \\
\hline & Frío & Caliente & Frío & Caliente \\
\hline Debajo de la media & 38 & 23 & 58 & 35 \\
\hline Arriba de la media & 38 & 9 & 45 & 20 \\
\hline \multirow{2}{*}{ BRONQUITIS } & \multicolumn{2}{|c|}{ 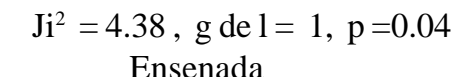 } & \multicolumn{2}{|c|}{$\begin{array}{c}\mathrm{Ji}^{2}=0.79, \text { g de } \mathrm{l}=1, \mathrm{p}=0.37 \\
\text { Guadalajara }\end{array}$} \\
\hline & Caliente & Caliente & \multicolumn{2}{|c|}{ Frío $\quad$ Caliente } \\
\hline Debajo de la media & 37 & 13 & 54 & 26 \\
\hline Arriba de la media & 35 & 13 & 57 & 18 \\
\hline \multirow[t]{2}{*}{ CATARRO COMÚN } & \multicolumn{2}{|c|}{$\begin{array}{c}\mathrm{Ji}^{2}=\underset{\text { Ensenada }}{0.06}, \mathrm{~g} \mathrm{de} \mathrm{l}=1, \mathrm{p}=0.79 \\
\text {. }\end{array}$} & \multicolumn{2}{|c|}{$\begin{array}{c}\mathrm{Ji}^{2}= \\
\text { Guadalajara }\end{array}$} \\
\hline & Frío & Caliente & Frío & Caliente \\
\hline Debajo de la media & 45 & 12 & 69 & 18 \\
\hline Arriba de la media & 40 & 12 & 52 & 18 \\
\hline \multirow[t]{2}{*}{ ASMA } & \multicolumn{2}{|c|}{$\mathrm{Ji}^{2}=0.65$, g de $\mathrm{l}=1, \mathrm{p}=0.79$} & \multicolumn{2}{|c|}{$\begin{array}{c}\mathrm{Ji}^{2}=0.55, \text { g de } \mathrm{l}=1, \mathrm{p}=0.45 \\
\text { Guadalajara }\end{array}$} \\
\hline & Frío & Caliente & Frío & Caliente \\
\hline Debajo de la media & 23 & 17 & 46 & 24 \\
\hline Arriba de la media & 30 & 11 & 47 & 14 \\
\hline \multirow[t]{2}{*}{ NEUMONÍA } & \multicolumn{2}{|c|}{$\mathrm{Ji}^{2}=\underset{\text { Ensenada }}{2.19, \quad \mathrm{~g} \text { de } \mathrm{l}=1, \mathrm{p}=0.13}$} & \multicolumn{2}{|c|}{$\begin{array}{c}\mathrm{Ji}^{2}=2.03, \text { g de } \mathrm{l}=1, \mathrm{p}=0.15 \\
\text { Guadalajara }\end{array}$} \\
\hline & Frío & Caliente & Frío & Caliente \\
\hline Debajo de la media & 37 & 15 & 52 & 30 \\
\hline Arriba de la media & 36 & 11 & 53 & 17 \\
\hline \multirow[t]{2}{*}{ TUBERCULOSIS } & \multicolumn{2}{|c|}{$\begin{array}{c}\mathrm{Ji}^{2}= \\
\text { Ensenada }\end{array}$} & \multicolumn{2}{|c|}{$\begin{array}{c}\mathrm{Ji}^{2}=2.67, \mathrm{~g} \mathrm{de} \mathrm{l}=1, \mathrm{p}=0.10 \\
\text { Guadalajara }\end{array}$} \\
\hline & Frío & Caliente & Frío & Caliente \\
\hline Debajo de la media & 27 & 13 & 41 & 35 \\
\hline \multirow[t]{2}{*}{ Arriba de la media } & \multirow{2}{*}{\multicolumn{2}{|c|}{$\mathrm{Ji}^{2}=0.13, \mathrm{gde} \mathrm{l}=1, \mathrm{p}=0.71$}} & & \\
\hline & & & \multicolumn{2}{|c|}{$\mathrm{Ji}^{2}=2.61, \mathrm{~g} \mathrm{del}=1, \mathrm{p}=0.10$} \\
\hline
\end{tabular}

Fuente: Cuestionario semi- estructurado

investigadas, en las personas muestreadas de estratos populares que asisten a los centros de salud estudiados, la falta de diferencias significativas en los porcentajes entre los grupos de Guadalajara y Ensenada (con sus dos excepciones), a pesar de la diferencia promedio de edad entre ambos, sugieren con firmeza, la hipótesis de la existencia de un sistema de conocimiento cultural compartido $(26,27)$.

Al proponer como definitiva la hipótesis de compartición espacial en el conocimiento, es decir, del acuerdo en el conocimiento humoral de las seis enfermedades respiratorias, entre los grupos de Guadalajara y Ensenada, podemos justificadamente pasar a discutir un segundo supuesto, que señala, que a pesar de que el varón y la mujer comparten el Revista Biomédica mismo conocimiento cultural, la mujer ha probado tener más elementos de conocimiento compartido que el hombre. Esta aseveración está respaldada por diversos estudios etnográficos, donde se señala, que la mujer tiene un mayor aprendizaje cultural (28) y que las mujeres mexicanas aplican sus conocimientos culturales, como cuidadoras de la salud doméstica (29-35). En otras palabras, es en la práctica cotidiana donde las mujeres mexicanas de estratos populares, comparten más y con mejor consistencia, que su contraparte masculina, los conocimientos culturales relacionados al proceso salud-enfermedad; esto concuerda con nuestra definición de conocimiento cultural que incluye las numerosas maneras de entender las causas, las implicaciones y los tratamientos de los 
Sistema frío/calor en los pacientes con afecciones respiratorias.

Cuadro 8

Cualidad humoral de padecimientos respiratorios seleccionados por años de escolaridad

\begin{tabular}{|c|c|c|c|c|}
\hline \multirow[t]{2}{*}{ GRIPE } & \multicolumn{2}{|c|}{ Ensenada } & \multicolumn{2}{|c|}{ Guadalajara } \\
\hline & Frío & Caliente & Frío & Caliente \\
\hline 3 años o menos & 15 & 12 & 43 & 14 \\
\hline 4 a 6 años & 24 & 6 & 41 & 19 \\
\hline 7 o más años & 37 & 14 & 19 & 13 \\
\hline \multirow{3}{*}{ BRONQUITIS } & \multicolumn{2}{|c|}{$\mathrm{Ji}^{2}=4.29, \mathrm{~g} \mathrm{de} \mathrm{l}=2, \mathrm{p}=0.11$} & \multicolumn{2}{|c|}{$\mathrm{Ji}^{2}=2.51, \mathrm{~g}$ de $\mathrm{l}=2, \mathrm{p}=0.28$} \\
\hline & \multicolumn{2}{|c|}{ Ensenada } & \multicolumn{2}{|c|}{ Guadalajara } \\
\hline & Frío & Caliente & Frío & Caliente \\
\hline 3 años o menos & 12 & 13 & 49 & 16 \\
\hline 4 a 6 años & 22 & 5 & 43 & 19 \\
\hline 7 o más años & 38 & 8 & 19 & 9 \\
\hline \multirow[t]{2}{*}{ CATARRO COMÚN } & \multicolumn{2}{|c|}{$\mathrm{Ji}^{2}=11.18, \mathrm{~g}$ de $\mathrm{l}=2, \mathrm{p}=0.03$} & \multicolumn{2}{|c|}{$\begin{array}{c}\mathrm{Ji}^{2}=0.80, \text { g de } \mathrm{l}=2, \mathrm{p}=0.66 \\
\text { Guadalajara }\end{array}$} \\
\hline & Frío & Caliente & \multicolumn{2}{|c|}{ Erío Guadalajara } \\
\hline 3 años o menos & 16 & 10 & 53 & 14 \\
\hline 4 a 6 años & 26 & 6 & 48 & 11 \\
\hline 7 o más años & 43 & 8 & 20 & 11 \\
\hline \multirow[t]{2}{*}{ ASMA } & \multicolumn{2}{|c|}{$\mathrm{Ji}^{2}=5.48, \mathrm{~g} \mathrm{de} \mathrm{l}=2, \mathrm{p}=0.06$} & \multicolumn{2}{|c|}{$\begin{array}{c}\mathrm{Ji}^{2}=3.53, \quad \mathrm{~g} \mathrm{de} \mathrm{l}=2, \mathrm{p}=0.17 \\
\text { Guadalajara }\end{array}$} \\
\hline & Frío & Caliente & \multicolumn{2}{|c|}{$\begin{array}{l}\text { Guadalajara } \\
\text { Frío }\end{array}$} \\
\hline 3 años o menos & 15 & 9 & 410 & 16 \\
\hline 4 a 6 años & 11 & 8 & 39 & 13 \\
\hline 7 o más años & 27 & 11 & 13 & 9 \\
\hline \multirow{2}{*}{ NEUMONIA } & \multicolumn{2}{|c|}{$\mathrm{Ji}^{2}=\underset{\text { Ensenada }}{1.10,}$ g de $\mathrm{l}=2, \mathrm{p}=0.57$} & \multicolumn{2}{|c|}{$\begin{array}{c}\mathrm{Ji}^{2}=1.94, \quad \mathrm{~g} \mathrm{de} \mathrm{l}=2, \mathrm{p}=0.37 \\
\text { Guadalajara }\end{array}$} \\
\hline & Frío & Caliente & Frío & Caliente \\
\hline 3 años o menos & 12 & 9 & 49 & 16 \\
\hline 4 a 6 años & 23 & 7 & 40 & 19 \\
\hline 7 o más años & 38 & 10 & 16 & 12 \\
\hline \multirow[t]{2}{*}{ TUBERCULOSIS } & \multicolumn{2}{|c|}{$\mathrm{Ji}^{2}=\underset{\text { Ensenada }}{3.85, \quad \mathrm{~g} \text { de } \mathrm{l}=2, \mathrm{p}=0.14}$} & \multicolumn{2}{|c|}{$\begin{array}{c}\mathrm{Ji}^{2}=3.12, \mathrm{~g} \text { de } \mathrm{l}=2, \mathrm{p}=0.20 \\
\text { Guadalajara }\end{array}$} \\
\hline & Frío & Caliente & Frío & Caliente \\
\hline 3 años o menos & 11 & 11 & 27 & 15 \\
\hline 4 a 6 años & 16 & 5 & 33 & 21 \\
\hline \multirow[t]{2}{*}{7 o más años } & & 7 & 12 & 13 \\
\hline & \multicolumn{2}{|c|}{$\mathrm{Ji}^{2}=5.50, \mathrm{~g} \mathrm{de} \mathrm{l}=2, \mathrm{p}=0.06$} & \multicolumn{2}{|c|}{$\mathrm{Ji}^{2}=1.83, \mathrm{~g}$ de $1=2, \mathrm{p}=0.40$} \\
\hline
\end{tabular}

Fuente: Cuestionario semi- estructurado

padecimientos respiratorios, que resultan de la experiencia cotidiana, independientemente de su aceptabilidad biomédica.

Al encontrar mayores porcentajes y significancia estadística en la diferencias sobre el acuerdo de la cualidad humoral frío/calor, a favor de las mujeres versus los varones, la sugerencia hipotética sobre la diferencia de mayor porcentaje de conocimiento cultural compartido en el género femenino, recibe un fuerte y claro impulso, sobre todo por sus implicaciones en las acciones e intervenciones educativas y de prácticas relacionadas con problemas respiratorios.

A diferencia de la asociación estadísticamente significativa entre el papel femenino y el mayor porcentaje de respuestas afirmativas acerca del carácter frío de los problemas respiratorios analizados; para nuestra sorpresa, no fue así cuando relacionamos conocimiento humoral con la escolaridad y la edad de las personas estudiadas, poniendo de manifiesto 


\section{JE García de Alba G, C von Glascoe, AJ Rubel, CC Moore, S Casasola, M Marquez-Amezcua.}

como el factor cultural traspasa estas variables, es decir, que independientemente de la edad y la escolaridad intragrupos; la condición humoral frío/ calor se encuentra enraizada en el conocimiento cultural sobre padecimientos respiratorios en los estratos populares que acuden a los centros de salud estudiados.

El mayor porcentaje de conocimientos culturales sobre la calidad humoral fría de las enfermedades investigadas, muestra la importancia del género como categoría interviniente en el proceso salud-enfermedad-atención. Decimos esto, debido a que frecuentemente se maneja de manera implícita, el supuesto de que ciertas variables como la edad y la escolaridad, por sí solas, otorgan ventaja para el desarrollo y cambio de actitudes y conductas positivas para la salud de las personas.

Quizás este supuesto de la edad y la escolaridad sea plausible para explicar causalmente acciones referentes al nivel individual en ciertos casos (36), pero a nivel del ámbito social, estas variables (escolaridad y edad) están interconectadas con otros aspectos como son la autonomía y la autoridad (37) que incrementan la variabilidad intracultural de manera amplia y a la vez específica de acuerdo a las determinaciones socio-económicas.

Lo anterior nos lleva a considerar la importancia teórica y práctica de las diferencias entre la escolaridad y la aculturación así como la edad y la generación de los pacientes.

Por eso, cuando cambian las circunstancias, cuando se vive la experiencia de una enfermedad respiratoria de impacto social, estas categorías y variables cobran relevancia. Tal es el caso de la tuberculosis, donde la relación abandono del tratamiento y el nivel de escolaridad reportan asociación estadísticamente significativa, a diferencia de la edad que no presenta asociación estadística con la adherencia al tratamiento (38), resultados que muestran no tan solo la variabilidad cultural (39), sino también la importancia del contexto social y económico de los pacientes.

La importancia sanitaria de las dimensiones analizadas, se manifiesta por su complejidad. Sobre todo si tomamos en cuenta que la única enfermedad donde hubo desacuerdo entre géneros, respecto a su condición humoral, fue la tuberculosis, padecimiento que no tan sólo en México, sino también en Latinoamérica, se considera como un proceso grave, $(40,41)$, provocado según el saber popular por el trabajo duro y la falta de alimentación, reconociéndose el padecimiento, sólo hasta que la tos crónica, el "decaimiento" y el "enflaquecimiento" general (42) son ostensibles, y/o cuando se manifiesta la imagen clásica del paciente con hemoptisis, precisamente durante $o$ ya pasada la fase de alta contagiosidad.

En la representación popular sobre los cuidados respiratorios, desde el paciente con catarro o gripa, hasta el paciente con tuberculosis, se incluye la administración de remedios caseros, medicamentos y otras medidas. En estas representaciones destaca el rol de género, al constatar que los tratamientos tradicionales recomendados: el reposo, el abrigo y la alimentación fortificante como el caldo de pollo, ponche, agua de borraja, etc.(17), los proveen básicamente las mujeres del estrato popular estudiado.

Para finalizar, manifestamos la conveniencia de profundizar este tipo de estudios, para estructurar un conocimiento más completo del punto de vista del paciente en los diferentes ámbitos de atención del sector salud. Así por ejemplo, se pueden continuar haciendo comparaciones sobre la distribución del conocimiento humoral entre diversos grupos sociales (7) o analizar aspectos puntuales de este conocimiento humoral frío/calor, en función de otras variables como el número y tipo de convivientes, etc. Lo anterior ayudará a clarificar la relación Salud-Cultura y sociedad, bajo enfoques diversos como el género, generación y su influencia cognitiva en un mismo grupo social.

Los estudios antropológicos que demuestran la variabilidad cultural como respuesta grupal ante el proceso salud-enfermedad-atención, contribuyen a la eficacia aplicativa de las disciplinas fundamentales de la Salud Pública, la Epidemiología y la Administración en la prevención y control de las enfermedades respiratorias, donde se incluye la re-emergente tuberculosis, que precisamente da inicio aparentando una simple afección respiratoria (43) producto que el

\section{Revista Biomédica}




\section{Sistema frío/calor en los pacientes con afecciones respiratorias.}

conocimiento popular de este tipo de pacientes, atañe a un desequilibrio del sub sistema frío/calor.

\section{REFERENCIAS.}

1.- Rubel A, O`Neill c y Collado R. Introducción al susto. En: Campos R. (comp) La antropología médica en México. Tomo 2. México: Instituto Mora/UAM; 1992. p 107.

2.- OMS. Informe sobre la salud en el mundo 2002. Ginebra: OMS; 2002. p. 196.

3.- OPS. La Salud en las Américas. Vol. II. Washington. DC. 2002. Publicación científica No 587. p. 433.

4.- Secretaria de Salud (SSA). Modificación a la norma oficial mexicana NOM-006-SSA2-1993, para la prevención y control de la tuberculosis en la atención primaria a la salud. México: 23 de Junio del 2000.

5.- Vargas LA. An anthropological look at disease and suffering. Gac Med Mex 1991; 127: 3-5.

6.- Baer R, Weller SC, Patcher L, Trotter R, García de Alba J, Glazer M, et al. Cross Cultural Perspectives on the common cold: data from five populations. Human Organization 1999; 3: 251-60.

7.- Menegoni L. Conceptions of tuberculosis and therapeutics choices in highland Chiapas. México. Med Anthropol Quart 1996; 3: 381-401.

8.- Nichter M. Illness Semantics and International health: The weak lungs/TB Complex in the Philippines. Soc Science Med 1994; 5: 649-63.

9.- Foster G. "Hippocrates' Latin American Legacy: Humoral Medicine in the New World" Langhorne. Gordon \& Breach; 1994.

10.- Kleiman A. Patients and healers in the context of culture. Berkeley: University of California Press; 1980. p. 138-45.

11.- Weller S, Rommey AK. Systematic data collection. Qualitative Research Methods. Vol 10. Newbury Park: Sage Publications; 1988.

12.- Bernard HR. Research methods in cultural anthropology. Newbury Park: Sage Publications; 1988. p. 29-61.

13.- Von Glascoe C, Metzger D. Enfrentando la amenaza de la tuberculosis en la frontera México-estadounidense. En:
Racionalidades y Transacciones en los Procesos de Cooperación Interna en salud en la Frontera Norte de México. El Colegio de la Frontera Norte.2000.

14.- Schartz N. Siento que me ahogo: Childhood asthma, medical pluralism and the Mexican frontier. [Doctoral Dissertation] San Francisco. University of California; 2001.

15.- Eggan F. Social anthropology and method of cross cultural controlled comparison. American Anthropologist 1954; 3: 743-61.

16.- Nader L. "Comparative Consciousness". In Assessing Cultural Anthropology. R Borofky (Ed). New York: Mac Graw - Hill Inc; 1994. p. 84 - 96.

17.- Estrella. E. Medicina Aborigen. Quito: Editorial Epoca; 1976. p. 77-9 y 165.

18.- López AA. La polémica frío/calor. Cuerpo Humano e ideología. Las concepciones de los antiguos nahuas. Instituto de Investigaciones Antropológicas, México: UNAM; 1980. Vol. 2. p. 303-18.

19.- Kay M. Health and illness in a Mexican barrio. In E.H. Spicer Ed. Ethnic Medicine in the Southwest. Tucson: University of Arizona Press; 1999. p. 99-161.

20.- Logan MH. Humoral medicine in Guatemala and peasant acceptance of modern medicine. In Health and Human Condition: Perspectives on Medical anthropology. MH Logan, EE Hunt. Jr; eds. North Situate: Duxbury Press; 1978 p. 363-75.

21.- Weller S. New data on intracultural variability: the hot cold concept of medicine and illness. Human Organization. 1983; 3: $249-56$.

22.- Bastien SN. Cognitive patterns of continuity in andeans studies in Past and present in the Americas. 44 th International Congress of Americanist; 1982. p. 139-45.

23.- Ackerkenecht E. Säftelehre einst un jetz. Curare 1984; (7). p. 111-6.

24.- Hahold A. El sistema cálido-fresco en la región sur andina del Perú. Aspectos de una clasificación popular de enfermedades hierbas medicinales y alimentos. En: A Kroeger y W Ruiz Cano (comps). Conceptos y tratamientos populares de algunas enfermedades en Latinoamérica. Cuzco. Perú; 1988. p. 39.

25.- Kuschick I. Medicina Popular en España. Madrid: Siglo 


\section{JE García de Alba G, C von Glascoe, AJ Rubel, CC Moore, S Casasola, M Marquez-Amezcua.}

XXI Editores; 1995. p. 80-3.

26.- Boster J. Exchange of varieties and information between aguaruma manioc cultivators. American Anthropologist 1986; 2: 429-36.

27.- Romney AK, Weller SC, Batchelder WH. Culture as consensus: a theory of cultural informants accuracy. American Anthropologist 1986; 2: 313-38.

28.- Garro LC. Intracultural variation in folk medical knowledge: A comparison between curers and noncurers. American Anthropologist 1986; 2: 351-70.

29.- Browner $\mathrm{CH}$. Gender politics in the distribution of therapeutic herbal knowledge. Med Anthropol Quart 1991; 1:99-132.

30.- Browner CH, Perdue ST. Women's secrets: bases for reproductive and social autonomy in a Mexican community. American Ethnologist 1988; 1: 84-97.

31.- Huber BR, Anderson R. Bonesetters and curers in a mexican community: conceptual models, status and gender. Med Anthropol 1990; 1: 23-38.

32.- Witheford MB. The patient as diagnostician: intracultural differences in illness etiology in a Mexican neighborhood. J Develop Societies 1991; 7: 256-68.

33.- Young J. Medical choice in a Mexican village. New Brunswick: Rutgers University Press; 1981.

34.- Zolla C. Medicina Tradicional y Enfermedad. México: Centro Interamericano de Estudios de Seguridad Social. 1988. p. 11-32.

35.- Grupo de Trabajo sobre Salud y Culturas Médicas Tradicionales en América Latina y el Caribe. Washington: Organización Panamericana de la Salud; 1983.

36.- Mac Mahon B, Trichoupulos D. Epidemiología 2da Edición. Madrid: Marban; 2001. p. 19-29.

37.- Loustanau MO, Sobo EJ. The context of health, illness and medicine. Wesport: CT Bergin \& Garvay; 1997. p. 63.

38.- Mejía MJ, Carmona GE. Abandono del tratamiento antituberculoso factores asociados. Rev Med IMSS (Méx.) 1995; 5: 481-5.

39.- Ostrosky-Zeichner L, Rangel FMS, García RE, Vázquez A, Ibarra MJ, Ponce de León RS. Tuberculosis en

\section{Revista Biomédica}

trabajadores de la salud: importancia de los programas de vigilancia y control. Salud Pública Mex 2000; 42: 48-52.

40.- García GML, Valdespino GJL, Palacios MM, García SC, Sepúlveda AJ. Tuberculosis y SIDA en México. Salud Pública de Mex 1995; 6 : 539-48.

41.- Poss JE. The meaning of tuberculosis for Mexican migrants farm workers in the United States. Social Science Med 1998; 2: 195-202.

42.- Rubel AJ. Seminario de Antropología Médica. Unidad de Investigación Social, Epidemiológica y de Servicios de Salud. Instituto Mexicano del Seguro Social. Guadalajara, Jalisco. México. 18 -22 Sept. 2000.

43.- Alvarez -Gordillo GC, Alvarez -Gordillo JF, Dorantes Jimenez JE, Halperin-Frisch D. Percepciones y prácticas relacionadas con la tuberculosis y la adherencia al tratamiento en Chiapas, México. Salud Pública Mex 2000; 42: 520-28. 